\title{
Nonlinear Approximation in the Large Deviations Principle
}

\author{
O. A. Yarova *, Ya. I. Yeleyko \\ Department of Theoretical and Applied Statistics, Ivan Franko National University of Lviv, Ukraine
}

\begin{abstract}
The Markov random processes and their approximations are considered. The main object of study is the exponential generator of random processes with independent increments, which are the solution of the problems of large deviations. These processes satisfy the conditions that make it possible to consider the Poisson and Lévy approximation. Generators of random processes are normalized by nonlinear parameters. Found explicit form of normalization parameter estimation.
\end{abstract}

Keywords Markov random processes and their approximations

AMS 2010 subject classifications. 60G25, 60G35

DOI: $10.19139 /$ soic.v6i4.317

\section{Introduction}

The problem of large deviations was originated as a method of solving statistical problems associated with the estimation of probability of rare events. The first work in this direction was the article by Cramér [1], but ultimately the method was developed in the article by Chernoff [2]. Publications [3, 4] are also related to this problem. The purpose of solving the problem of large deviations is finding of action functional

$$
I(x)=I\left(x_{0}\right)+\int_{0}^{\infty} L\left(x(s), x^{\prime}(s)\right) d s,
$$

where $x(s)$ is a Markov process, function $L(x, u)$ defined by the exponential generator

$$
L(x, u)=\sup _{p \in R}\{p u-H(v, p)\},
$$

where $p:=\varphi^{\prime}(u), H\left(v, \varphi^{\prime}(u)\right):=H_{\Gamma} \varphi(u)$. In the writing [5] in the scheme of Poisson approximation processes with independent increments without diffusion component were considered. Between jumps there were Markov processes with linear normalizing factor.

These processes are defined by the generator

$$
\Gamma_{\varepsilon}^{\delta} \varphi(u)=\varepsilon^{-1} \int_{R}(\varphi(u+\varepsilon v)-\varphi(u)) \Gamma^{\delta}(d v) .
$$

in the scale of time $t / \varepsilon$.

In the articles [7]-[9] the generator of Markov process and their evolutions were considered in the scale of time $t / g_{1}(\varepsilon)$ and $t / g_{2}(\varepsilon)$ in the Poisson and Lévi approximation.

\footnotetext{
*Correspondence to: Oksana Yarova (Email: Moklyachuk@gmail.com). Department of Theoretical and Applied Statistics, Ivan Franko National University of Lviv,1, University str. Lviv 79000 Ukraine
}

ISSN 2310-5070 (online) ISSN 2311-004X (print)

Copyright (C) 2018 International Academic Press 
The purpose of this work is to find functions that normalize the generator of random process with independent increments in the scale of time $t / g_{2}(\varepsilon)$ in the Large Deviations Principle.

These processes are defined by the generator

$$
\Gamma^{\varepsilon} \varphi(u)=\left(g_{2}(\varepsilon)\right)^{-1} \int_{R}\left(\varphi\left(u+g_{1}(\varepsilon) v\right)-\varphi(u)\right) \Gamma^{\varepsilon}(d v) .
$$

Besides this, in the Poisson approximation the next condition take places

$$
\left(g_{2}(\varepsilon)\right)^{-1} g_{1}(\varepsilon) f_{1}(\varepsilon) \rightarrow 1, \quad \varepsilon \rightarrow 0,
$$

and in the Lévy approximation

$$
\left(g_{3}(\varepsilon)\right)^{-1} g_{1}(\varepsilon) f_{2}(\varepsilon) \rightarrow 1, \quad \varepsilon \rightarrow 0,
$$

where $g_{1}(\varepsilon), g_{2}(\varepsilon), g_{3}(\varepsilon), f_{1}(\varepsilon), f_{2}(\varepsilon)$ are normalization function, such that $g_{2}(\varepsilon)=o\left(g_{1}(\varepsilon)\right), g_{3}(\varepsilon)=o\left(g_{2}(\varepsilon)\right)$, $f_{2}(\varepsilon)=o\left(f_{1}(\varepsilon)\right), g_{1}(\varepsilon), g_{2}(\varepsilon), g_{3}(\varepsilon), f_{1}(\varepsilon), f_{2}(\varepsilon) \rightarrow 0, \varepsilon \rightarrow 0$.

\section{Poisson approximation}

We consider Markov processes with locally independent increments $\eta^{\varepsilon}(\cdot)$ with trajectories in $D^{R}[0, \infty)$ :

$$
\eta^{\varepsilon}=g_{1}(\varepsilon) \eta\left(\frac{t}{g_{2}(\varepsilon)}\right), \quad t \geq 0
$$

In this normalization $g_{1}(\varepsilon), g_{2}(\varepsilon) \rightarrow 0$ as $\varepsilon \rightarrow 0$.

These processes are defined by the generator

$$
\Gamma^{\varepsilon} \varphi(u)=\left(g_{2}(\varepsilon)\right)^{-1} \int_{R}\left(\varphi\left(u+g_{1}(\varepsilon) v\right)-\varphi(u)\right) \Gamma^{\varepsilon}(d v),
$$

where $\varphi(u)$ is a twice differentiable function on $R$ which tends to 0 at infinity and with sup-norm, $\varphi(u) \in C_{0}^{2}(R)$. The kernel of intensity $\Gamma^{\varepsilon}$ belongs to the class $C^{3}(R)$. This kernel satisfies the condition $\Gamma^{\varepsilon}(0)=0$.

We consider the problem of large deviations in the scheme of Poisson approximation in the case where the following conditions are satisfied:

(P1) Approximation of the mean values:

$$
b_{\varepsilon}=\int_{R} v \Gamma^{\varepsilon}(d v)=f_{1}(\varepsilon)\left(b+\theta_{b}^{\varepsilon}\right)
$$

and

$$
c_{\varepsilon}=\int_{R} v^{2} \Gamma^{\varepsilon}(d v)=f_{1}(\varepsilon)\left(c+\theta_{c}^{\varepsilon}\right),
$$

where $b, c<\infty,\left|\theta_{b}^{\varepsilon}\right| \rightarrow 0,\left|\theta_{c}^{\varepsilon}\right| \rightarrow 0, f_{1}(\varepsilon) \rightarrow 0, \varepsilon \rightarrow 0$.

(P2) The Poisson approximation condition for the intensity kernel

$$
\Gamma_{q}^{\varepsilon}=\int_{R} q(v) \Gamma^{\varepsilon}(d v)=f_{1}(\varepsilon)\left(\Gamma_{q}+\theta_{q}^{\varepsilon}\right)
$$

for all $q(\cdot)$ from $C_{3}(R)$

The kernel $\Gamma_{q}$ has the following representation:

$$
\Gamma_{q}=\int_{R} q(v) \Gamma^{0}(d v) .
$$


Items $\theta_{b}^{\varepsilon}, \theta_{c}^{\varepsilon}, \theta_{q}^{\varepsilon}$ satisfied the conditions $\left|\theta^{\varepsilon}\right| \rightarrow 0, f_{1}(\varepsilon) \rightarrow 0$ as $\varepsilon \rightarrow 0$.

(P3) The limiting generator is without diffuse component

$$
c=\int_{R} v^{2} \Gamma^{0}(d v)=\text { const. }
$$

(P4) Square-integrability condition

$$
\lim _{c \rightarrow \infty} \int_{|v|>c} v^{2} \Gamma^{\varepsilon}(d v)=0
$$

(P5) The exponential boundedness

$$
\int_{R} e^{p|v|} \Gamma_{q}(d v)<\infty
$$

In the scheme of Poisson approximation the solution of the problem of large deviations for these processes are defined by nonlinear exponential generator:

$$
H_{\Gamma}^{\varepsilon} \varphi(u)=e^{-\frac{\varphi(u)}{g_{1}(\varepsilon)}} g_{1}(\varepsilon) \Gamma^{\varepsilon} e^{\frac{\varphi(u)}{g_{1}(\varepsilon)}} .
$$

\section{Lemma 1}

The exponential generator in the scheme of Poisson approximation has the following asymptotical representation

$$
H_{\Gamma}^{\varepsilon} \varphi(u)=H_{\Gamma} \varphi(u)+\theta_{\Gamma}^{\varepsilon} \varphi
$$

provided $\left(g_{2}(\varepsilon)\right)^{-1} g_{1}(\varepsilon) f_{1}(\varepsilon) \rightarrow 1$, where $\varphi(u)$ is from $C_{0}^{3}(R)$ and $\left|\theta_{\Gamma}^{\varepsilon} \varphi\right| \rightarrow 0$, as $g_{1}(\varepsilon), f_{1}(\varepsilon) \rightarrow 0$, as $\varepsilon \rightarrow 0$,

$$
H_{\Gamma} \varphi(u)=b \varphi^{\prime}(u)+\int_{R}\left(e^{v \varphi^{\prime}(u)}-1-v \varphi^{\prime}(u)\right) \Gamma^{0}(d v) .
$$

Proof

The generator of Markov processes has the next form

$$
\Gamma^{\varepsilon} \varphi(u)=\left(g_{2}(\varepsilon)\right)^{-1} \int_{R}\left(\varphi\left(u+g_{1}(\varepsilon) v\right)-\varphi(u)\right) \Gamma^{\varepsilon}(d v),
$$

so, for the exponential generator

$$
H_{\Gamma}^{\varepsilon} \varphi(u)=\left(g_{2}(\varepsilon)\right)^{-1} g_{1}(\varepsilon) \int_{R}\left(e^{\Delta_{\varepsilon} \varphi(u)}-1\right) \Gamma^{\varepsilon}(d v),
$$

where $\Delta_{\varepsilon} \varphi(u)=\left(g_{1}(\varepsilon)\right)^{-1}\left(\varphi\left(u+g_{1}(\varepsilon) v\right)-\varphi(u)\right)$.

We can write the generator in the form

$$
\begin{gathered}
H_{\Gamma}^{\varepsilon} \varphi(u)=\left(g_{2}(\varepsilon)\right)^{-1} g_{1}(\varepsilon) \int_{R}\left(e^{\Delta_{\varepsilon} \varphi(u)}-1-\Delta_{\varepsilon} \varphi(u)-\frac{1}{2}\left(\Delta_{\varepsilon} \varphi(u)\right)^{2}\right) \Gamma^{\varepsilon}(d v)+ \\
\left(g_{2}(\varepsilon)\right)^{-1} g_{1}(\varepsilon) \int_{R}\left(\Delta_{\varepsilon} \varphi(u)+\frac{1}{2}\left(\Delta_{\varepsilon} \varphi(u)\right)^{2}\right) \Gamma^{\varepsilon}(d v) .
\end{gathered}
$$

where the function $e^{\Delta_{\varepsilon} \varphi(u)}-1-\Delta_{\varepsilon} \varphi(u)-\frac{1}{2}\left(\Delta_{\varepsilon} \varphi(u)\right)^{2}$ is from $C_{3}(R)$, and since

$$
\frac{e^{\Delta_{\varepsilon} \varphi(u)}-1-\Delta_{\varepsilon} \varphi(u)-\frac{1}{2}\left(\Delta_{\varepsilon} \varphi(u)\right)^{2}}{v^{2}} \rightarrow 0 \quad \text { as } v \rightarrow 0 .
$$

In addition, this function is continuous and bounded, because $\varphi(u)$ is from $C_{0}^{2}(R)$. So, from conditions $\mathbf{P 1}$ and P2 we obtain: 


$$
\begin{aligned}
& H_{\Gamma}^{\varepsilon} \varphi(u)=\left(g_{2}(\varepsilon)\right)^{-1} g_{1}(\varepsilon) f_{1}(\varepsilon) \int_{R}\left(e^{\Delta_{\varepsilon} \varphi(u)}-1-\Delta_{\varepsilon} \varphi(u)-\frac{1}{2}\left(\Delta_{\varepsilon} \varphi(u)\right)^{2}\right) \Gamma^{0}(d v)+ \\
&\left(g_{2}(\varepsilon)\right)^{-1} g_{1}(\varepsilon) \int_{R}\left(\Delta_{\varepsilon} \varphi(u)-v \varphi(u)-g_{1}(\varepsilon) \frac{v^{2}}{2} \varphi^{\prime \prime}(u)\right) \Gamma^{\varepsilon}(d v)+ \\
&\left(g_{2}(\varepsilon)\right)^{-1} g_{1}(\varepsilon) f_{1}(\varepsilon) b \varphi^{\prime}(u)+\frac{1}{2}\left(g_{2}(\varepsilon)\right)^{-1}\left(g_{1}(\varepsilon)\right)^{2} f_{1}(\varepsilon) c \varphi^{\prime \prime}(u)+ \\
&\left(g_{2}(\varepsilon)\right)^{-1} g_{1}(\varepsilon) \int_{R}\left(\frac{1}{2}\left(\Delta_{\varepsilon} \varphi(u)\right)^{2}-\frac{v^{2}}{2}\left(\varphi^{\prime}(u)\right)^{2}\right) \Gamma^{\varepsilon}(d v)+ \\
&\left(g_{2}(\varepsilon)\right)^{-1} g_{1}(\varepsilon) f_{1}(\varepsilon) \frac{1}{2} c\left(\varphi^{\prime}(u)\right)^{2} .
\end{aligned}
$$

Applying the Taylor's formula for $\varphi(u)$ and making use of the condition P2:

$$
\begin{gathered}
H_{\Gamma}^{\varepsilon} \varphi(u)=\left(g_{2}(\varepsilon)\right)^{-1} g_{1}(\varepsilon) f_{1}(\varepsilon) \int_{R}\left(e^{v \varphi^{\prime}(u)}-1-v \varphi^{\prime}(u)-\frac{v^{2}}{2}\left(\varphi^{\prime}(u)\right)^{2}\right) \Gamma^{0}(d v)+ \\
\left(g_{2}(\varepsilon)\right)^{-1} g_{1}(\varepsilon) f_{1}(\varepsilon) \int_{R}\left(e^{v \varphi^{\prime}(u)} g_{1}(\varepsilon) \frac{v^{2}}{2} \varphi^{\prime \prime}(\tilde{u})-g_{1}(\varepsilon) \frac{v^{2}}{2} \varphi^{\prime \prime}(\tilde{u})-\right. \\
\left.g_{1}(\varepsilon) \frac{v^{4}}{8}\left(\varphi^{\prime \prime}(\tilde{u})\right)^{2}\right) \Gamma^{0}(d v)+ \\
\left(g_{2}(\varepsilon)\right)^{-1} g_{1}(\varepsilon) f_{1}(\varepsilon) \int_{R} g_{1}(\varepsilon) \frac{v^{3}}{3 !} \varphi^{\prime \prime \prime}(\tilde{u}) \Gamma^{0}(d v)+ \\
\left(g_{2}(\varepsilon)\right)^{-1} g_{1}(\varepsilon) f_{1}(\varepsilon) b \varphi^{\prime}(u)+\frac{1}{2} g_{1}\left(g_{2}(\varepsilon)\right)^{-1}\left(g_{1}(\varepsilon)\right)^{2} f_{1}(\varepsilon) c \varphi^{\prime \prime}(\tilde{u})+ \\
\left(g_{2}(\varepsilon)\right)^{-1} g_{1}(\varepsilon) f_{1}(\varepsilon) \int_{R} g_{1}(\varepsilon) \frac{v^{4}}{4}\left(\varphi^{\prime \prime}(\tilde{u})\right)^{2} \Gamma^{0}(d v)+ \\
\left(g_{2}(\varepsilon)\right)^{-1} g_{1}(\varepsilon) f_{1}(\varepsilon) c \frac{1}{2}\left(\varphi^{\prime}(u)\right)^{2} .
\end{gathered}
$$

From condition $\mathbf{P 3}$ and the condition $\left(g_{2}(\varepsilon)\right)^{-1} g_{1}(\varepsilon) f_{1}(\varepsilon) \rightarrow 1$ we obtain

$$
H_{\Gamma}^{\varepsilon} \varphi(u)=H_{\Gamma} \varphi(u)+\theta_{\Gamma}^{\varepsilon} \varphi
$$

where $\left|\theta_{\Gamma}^{\varepsilon} \varphi\right| \rightarrow 0$, as $g_{1}(\varepsilon), f_{1}(\varepsilon) \rightarrow 0$.

Lemma is proved.

From this Lemma we have that the following Theorem holds true.

\section{Theorem 1}

The solution for the problem of large deviations for the process

$$
\begin{gathered}
\eta^{\varepsilon}=g_{1}(\varepsilon)\left(\frac{t}{g_{2}(\varepsilon)}\right), \quad t \geq 0, \\
\Gamma^{\varepsilon} \varphi(u)=\left(g_{2}(\varepsilon)\right)^{-1} \int_{R}\left(\varphi\left(u+g_{1}(\varepsilon) v\right)-\varphi(u)\right) \Gamma^{\varepsilon}(d v)
\end{gathered}
$$


under conditions (P1-P5) and

$$
\left(g_{2}(\varepsilon)\right)^{-1} g_{1}(\varepsilon) f_{1}(\varepsilon) \rightarrow 1, \varepsilon \rightarrow 0,
$$

is determined by the limit generator $H_{\Gamma}$ of the form

$$
H_{\Gamma} \varphi(u)=b \varphi^{\prime}(u)+\int_{R}\left(e^{v \varphi^{\prime}(u)}-1-v \varphi^{\prime}(u)\right) \Gamma^{0}(d v) .
$$

Remark 1

Condition $\left(g_{2}(\varepsilon)\right)^{-1} g_{1}(\varepsilon) f_{1}(\varepsilon) \rightarrow 1$ can be satisfied not only for trivial functions $g_{2}(\varepsilon)=\varepsilon^{2}, g_{1}(\varepsilon)=\varepsilon, f_{1}(\varepsilon)=\varepsilon$, as in the article [6], but for more complex functions, such as $g_{2}(\varepsilon)=\varepsilon^{2}, g_{1}(\varepsilon)=\sin \varepsilon, f_{1}(\varepsilon)=\cos \varepsilon$.

\section{Lévy approximation}

Now consider another normalization for the family of Markov processes with trajectories in $D^{R}[0, \infty)$

$$
\eta^{\varepsilon}=g_{1}(\varepsilon) \eta\left(\frac{t}{g_{3}(\varepsilon)}\right), \quad t \geq 0
$$

In this normalization $g_{1}(\varepsilon), g_{3}(\varepsilon) \rightarrow 0$, as $\varepsilon \rightarrow 0$.

These processes are defined by generator

$$
\Gamma^{\varepsilon} \varphi(u)=\left(g_{3}(\varepsilon)\right)^{-1} \int_{R}\left(\varphi\left(u+g_{1}(\varepsilon) v\right)-\varphi(u)\right) \Gamma^{\varepsilon}(d v),
$$

where $\varphi(u)$ is a twice differentiable function on $R$ which tends to 0 at infinity and with sup-norm, $\varphi(u) \in C_{0}^{2}(R)$. The kernel of intensity $\Gamma^{\varepsilon}$ belongs to the class $C^{3}(R)$. This kernel satisfies the condition $\Gamma^{\varepsilon}(0)=0$.

The conditions of Lévy approximation are the following:

(L1) Approximation of the mean values:

$$
b_{\varepsilon}=\int_{R} v \Gamma^{\varepsilon}(d v)=f_{1}(\varepsilon) b_{1}+f_{2}(\varepsilon)\left(b+\theta_{b}^{\varepsilon}\right)
$$

and

$$
c_{\varepsilon}=\int_{R} v^{2} \Gamma^{\varepsilon}(d v)=f_{2}(\varepsilon)\left(c+\theta_{c}^{\varepsilon}\right)
$$

where $b<\infty, c<\infty,\left|\theta_{b}^{\varepsilon}\right| \rightarrow 0,\left|\theta_{c}^{\varepsilon}\right| \rightarrow 0, f_{1}(\varepsilon), f_{2}(\varepsilon) \rightarrow 0$. In this normalization $f_{2}(\varepsilon)=o\left(f_{1}(\varepsilon)\right)$.

(L2) Lévy approximation condition for the intensity kernel

$$
\Gamma_{q}^{\varepsilon}=\int_{R} q(v) \Gamma^{\varepsilon}(d v)=f_{2}(\varepsilon)\left(\Gamma_{q}+\theta_{q}^{\varepsilon}\right)
$$

for all $q \in C_{3}(R)$.

This kernel has the following representation:

$$
\Gamma_{q}=\int_{R} q(v) \Gamma^{0}(d v)
$$

Items $\theta_{b}^{\varepsilon}, \theta_{c}^{\varepsilon}, \theta_{q}^{\varepsilon}$ satisfied the conditions $\left|\theta^{\varepsilon}\right| \rightarrow 0, f_{1}(\varepsilon) \rightarrow 0$ as $\varepsilon \rightarrow 0$.

(L3) Square-integrability condition

$$
\lim _{c \rightarrow \infty} \int_{|v|>c} v^{2} \Gamma^{\varepsilon}(d v)=0 .
$$

(L5) The exponential boundedness

$$
\int_{R} e^{p|v|} \Gamma_{q}(d v)<\infty
$$




\section{Example 3.1}

Consider the example of a family of Markov processes $\alpha^{\varepsilon}$, for which

$$
\begin{gathered}
P\left\{\alpha^{\varepsilon}=f_{1}(\varepsilon) \alpha_{1}\right\}=p_{0}-f_{2}(\varepsilon) p_{1}, \\
P\left\{\alpha^{\varepsilon}=f_{2}(\varepsilon) \alpha\right\}=q_{0}, p_{0}+q_{0}=1, \\
P\left\{\alpha^{\varepsilon}=d\right\}=f_{2}(\varepsilon) p_{1} .
\end{gathered}
$$

Check the conditions of Lévy approximation.

Firstly, find the first and the second moments for the family of Markov processes

$$
\begin{gathered}
b_{\varepsilon}=E \alpha^{\varepsilon}=p_{0} f_{1}(\varepsilon) \alpha_{1}-f_{1}(\varepsilon) f_{2}(\varepsilon) \alpha_{1} p_{1}+q_{0} f_{2}(\varepsilon) \alpha+f_{2}(\varepsilon) p_{1} d= \\
f_{1}(\varepsilon)\left(p_{0} \alpha_{1}\right)+f_{2}(\varepsilon)\left(\alpha q_{0}+p_{1} d\right)+o\left(f^{2}(\varepsilon)\right) . \\
c_{\varepsilon}=E\left(\alpha^{\varepsilon}\right)^{2}=f_{1}^{2}(\varepsilon) \alpha_{1}\left(p_{0}-f_{2}(\varepsilon) p_{1}\right)+f_{2}^{2}(\varepsilon) \alpha^{2} q_{0}+d^{2} f_{2}(\varepsilon) p_{1}= \\
f_{2}(\varepsilon)\left(p_{1} d^{2}\right)+o\left(f_{2}^{2}(\varepsilon)\right) .
\end{gathered}
$$

So, we have the next parameters for condition (L1):

$$
\begin{gathered}
b_{1}=p_{0} \alpha_{1}, \\
b=\alpha q_{0}+p_{1} d, \\
c=p_{1} d^{2} .
\end{gathered}
$$

Now, find the intensity kernel

$$
\Gamma_{q}^{\varepsilon}=q\left(f_{1}(\varepsilon) \alpha_{1}\right) p_{0}+q\left(f_{2}(\varepsilon) \alpha\right) q_{0}-q\left(f_{1}(\varepsilon) \alpha_{1}\right)\left(-f_{2} p_{1}\right)+q(d) f_{2}(\varepsilon) p_{1}=f_{2}(\varepsilon) q(d) p_{1} .
$$

These moments satisfied the condition of approximation of the mean values. So, $\Gamma_{q}=p_{1} d^{2}$.

Thus, we have all parameters for process $\alpha^{\varepsilon}$ in Lévi approximation.

\section{Lemma 2}

The exponential generator

$$
H_{\Gamma}^{\varepsilon} \varphi(u)=e^{-\frac{\varphi(u)}{g_{1}(\varepsilon)}} g_{1}(\varepsilon) \Gamma^{\varepsilon} e^{\frac{\varphi(u)}{g_{1}(\varepsilon)}}
$$

in the scheme of Lévy approximation has the next asymptotically representation

$$
H_{\Gamma}^{\varepsilon} \varphi(u)=\left(g_{3}(\varepsilon)\right)^{-1} g_{1}(\varepsilon) f_{1}(\varepsilon) b_{1} \varphi^{\prime}(u)+H_{\Gamma} \varphi(u)+\theta_{\Gamma}^{\varepsilon} \varphi
$$

with conditions $\left(g_{3}(\varepsilon)\right)^{-1} g_{1}(\varepsilon) f_{2}(\varepsilon) \rightarrow 1, \varepsilon \rightarrow 0$, where $\varphi(u)$ is from $C_{0}^{3}(R)$ and

$$
H_{\Gamma} \varphi(u)=\left(b-b_{0}\right) \varphi^{\prime}(u)+\frac{1}{2}\left(c-c_{0}\right)\left(\varphi^{\prime}(u)\right)^{2}+\int_{R}\left(e^{v \varphi^{\prime}(u)}-1\right) \Gamma^{0}(d v),
$$

$b_{0}=\int_{R} v \Gamma^{0}(d v), c_{0}=\int_{R} v^{2} \Gamma^{0}(d v)$.

The neglected item $\left|\theta_{\Gamma}^{\varepsilon} \varphi\right| \rightarrow 0$ as $g_{1}(\varepsilon) \rightarrow 0$. 
Proof

We can represent the exponential generator in the form

$$
H_{\Gamma}^{\varepsilon} \varphi(u)=\left(g_{3}(\varepsilon)\right)^{-1} g_{1}(\varepsilon) \int_{R}\left(e^{\Delta_{\varepsilon} \varphi(u)}-1\right) \Gamma^{\varepsilon}(d v),
$$

where

$\Delta_{\varepsilon} \varphi(u)=\left(g_{1}(\varepsilon)\right)^{-1}\left(\varphi\left(u+g_{1}(\varepsilon) v\right)-\varphi(u)\right)$.

Rewrite the generator:

$$
\begin{gathered}
H_{\Gamma}^{\varepsilon} \varphi(u)= \\
\left(g_{3}(\varepsilon)\right)^{-1} g_{1}(\varepsilon) \int_{R}\left(e^{\Delta_{\varepsilon} \varphi(u)}-1-\Delta_{\varepsilon} \varphi(u)-\frac{1}{2}\left(\Delta_{\varepsilon} \varphi(u)\right)^{2}\right) \Gamma^{\varepsilon}(d v)+ \\
\left(g_{3}(\varepsilon)\right)^{-1} g_{1}(\varepsilon) \int_{R}\left(\Delta_{\varepsilon} \varphi(u)+\frac{1}{2}\left(\Delta_{\varepsilon} \varphi(u)\right)^{2}\right) \Gamma^{\varepsilon}(d v) .
\end{gathered}
$$

Function $e^{\Delta_{\varepsilon} \varphi(u)}-1-\Delta_{\varepsilon} \varphi(u)-\frac{1}{2}\left(\Delta_{\varepsilon} \varphi(u)\right)^{2}$ belongs to $C_{3}(R)$, since

$$
\frac{e^{\Delta_{\varepsilon} \varphi(u)}-1-\Delta_{\varepsilon} \varphi(u)-\frac{1}{2}\left(\Delta_{\varepsilon} \varphi(u)\right)^{2}}{v^{2}} \rightarrow 0 \quad \text { as } v \rightarrow 0 .
$$

In addition, this function is continuous and bounded, since $\varphi(u)$ from $C_{0}^{2}(R)$.

So, from conditions (L1) and (L2) we obtaine

$$
\begin{gathered}
H_{\Gamma}^{\varepsilon} \varphi(u)= \\
\left(g_{3}(\varepsilon)\right)^{-1} g_{1}(\varepsilon) f_{2}(\varepsilon) \int_{R}\left(e^{\Delta_{\varepsilon} \varphi(u)}-1-\Delta_{\varepsilon} \varphi(u)-\frac{1}{2}\left(\Delta_{\varepsilon} \varphi(u)\right)^{2}\right) \Gamma^{0}(d v)+ \\
\left(g_{3}(\varepsilon)\right)^{-1} g_{1}(\varepsilon) \int_{R}\left(\Delta_{\varepsilon} \varphi(u)-v \varphi^{\prime}(u)-g_{1}(\varepsilon) \frac{v^{2}}{2} \varphi^{\prime \prime}(u)\right) \Gamma^{\varepsilon}(d v)+ \\
\left(g_{3}(\varepsilon)\right)^{-1} g_{1}(\varepsilon) f_{1}(\varepsilon) b_{1} \varphi^{\prime}(u)+\left(g_{2}(\varepsilon)\right)^{-1} g_{1}(\varepsilon) f_{2}(\varepsilon) b \varphi^{\prime}(u)+ \\
\left(g_{3}(\varepsilon)\right)^{-1} g_{1}^{2}(\varepsilon) f_{2}(\varepsilon) \frac{1}{2} c \varphi^{\prime \prime}(u)+ \\
\left(g_{3}(\varepsilon)\right)^{-1} g_{1}(\varepsilon) \int_{R}\left(\frac{1}{2}\left(\Delta_{\varepsilon} \varphi(u)\right)^{2}-\frac{v^{2}}{2}\left(\varphi^{\prime}(u)\right)^{2}\right) \Gamma^{\varepsilon}(d v)+ \\
\left(g_{3}(\varepsilon)\right)^{-1} g_{1}(\varepsilon) f_{2}(\varepsilon) \frac{1}{2} c\left(\varphi^{\prime}(u)\right)^{2} .
\end{gathered}
$$

Applying the Taylor's formula for $\varphi(u)$ and making use the condition $\mathbf{L} 2$ we will have

$$
\begin{gathered}
H_{\Gamma}^{\varepsilon} \varphi(u)= \\
\left(g_{3}(\varepsilon)\right)^{-1} g_{1}(\varepsilon) f_{2}(\varepsilon) \int_{R}\left(e^{v \varphi^{\prime}(u)}-1-v \varphi^{\prime}(u)-\frac{v^{2}}{2}\left(\varphi^{\prime}(u)\right)^{2}\right) \Gamma^{0}(d v)+ \\
\left(g_{3}(\varepsilon)\right)^{-1} g_{1}(\varepsilon) f_{2}(\varepsilon) \int_{R}\left(e^{v \varphi^{\prime}(u)} g_{1}(\varepsilon) \frac{v^{2}}{2} \varphi^{\prime \prime}(\tilde{u})-g_{1}(\varepsilon) \frac{v^{2}}{2} \varphi^{\prime \prime}(\tilde{u})-\right.
\end{gathered}
$$




$$
\begin{gathered}
\left.g_{1}(\varepsilon) \frac{v^{4}}{8}\left(\varphi^{\prime \prime}(\tilde{u})\right)^{2}\right) \Gamma^{0}(d v)+ \\
\left(g_{3}(\varepsilon)\right)^{-1} g_{1}(\varepsilon) f_{2}(\varepsilon) \int_{R} g_{1}(\varepsilon) \frac{v^{3}}{3 !} \varphi^{\prime \prime \prime}(\tilde{u}) \Gamma^{0}(d v)+\left(g_{3}(\varepsilon)\right)^{-1} g_{1}(\varepsilon) f_{1}(\varepsilon) b_{1} \varphi^{\prime}(u)+ \\
\left(g_{3}(\varepsilon)\right)^{-1} g_{1}(\varepsilon) f_{2}(\varepsilon) b \varphi^{\prime}(u)+\left(g_{3}(\varepsilon)\right)^{-1} g_{1}^{2}(\varepsilon) f_{2}(\varepsilon) \frac{1}{2} c \varphi^{\prime \prime}(\tilde{u})+ \\
\left(g_{3}(\varepsilon)\right)^{-1} g_{1}(\varepsilon) f_{2}(\varepsilon) \int_{R} g_{1}(\varepsilon) \frac{v^{4}}{4}\left(\varphi^{\prime \prime}(\tilde{u})\right)^{2} \Gamma^{0}(d v)+ \\
\left(g_{3}(\varepsilon)\right)^{-1} g_{1}(\varepsilon) f_{2}(\varepsilon) c \frac{1}{2}\left(\varphi^{\prime}(u)\right)^{2}
\end{gathered}
$$

Using condition (L3) and $\left(g_{3}(\varepsilon)\right)^{-1} g_{1}(\varepsilon) f_{2}(\varepsilon) \rightarrow 1$, finally we obtain

$$
H_{\Gamma}^{\varepsilon} \varphi(u)=\left(g_{3}(\varepsilon)\right)^{-1} g_{1}(\varepsilon) f_{1}(\varepsilon) b_{1} \varphi^{\prime}(u)+H_{\Gamma} \varphi(u)+\theta_{\Gamma}^{\varepsilon} \varphi,
$$

where $\left|\theta_{\Gamma}^{\varepsilon} \varphi\right| \rightarrow 0$ as $g_{1}(\varepsilon), f_{1}(\varepsilon) \rightarrow 0$.

Lemma is proved.

From this Lemma we have that the following Theorem holds true.

\section{Theorem 2}

The solution for the problem of large deviations for the process

$$
\begin{gathered}
\eta^{\varepsilon}=g_{1}(\varepsilon)\left(\frac{t}{g_{3}(\varepsilon)}\right), \quad t \geq 0, \\
\Gamma^{\varepsilon} \varphi(u)=\left(g_{3}(\varepsilon)\right)^{-1} \int_{R}\left(\varphi\left(u+g_{1}(\varepsilon) v\right)-\varphi(u)\right) \Gamma^{\varepsilon}(d v)
\end{gathered}
$$

under conditions (L1-L5) and

$$
\left(g_{3}(\varepsilon)\right)^{-1} g_{1}(\varepsilon) f_{2}(\varepsilon) \rightarrow 1, \varepsilon \rightarrow 0
$$

is determined by the limit generator $H_{\Gamma}$ of the form

$$
H_{\Gamma} \varphi(u)=\left(b-b_{0}\right) \varphi^{\prime}(u)+\frac{1}{2}\left(c-c_{0}\right)\left(\varphi^{\prime}(u)\right)^{2}+\int_{R}\left(e^{v \varphi^{\prime}(u)}-1\right) \Gamma^{0}(d v) .
$$

\section{Conclusions.}

Thus, using normalization parameters as nonlinear functions we can found asymptotic representation for the random process in Poisson and Lévy approximation. This normalization allows to find nonlinear exponential generator that is the solution of the Large Deviations Principle.As normalization factors, we can take not only $\varepsilon$, but other nonlinear functions $f(\varepsilon)$ and $g(\varepsilon)$, that satisfied the conditions

$$
\left(g_{2}(\varepsilon)\right)^{-1} g_{1}(\varepsilon) f_{1}(\varepsilon) \rightarrow 1, \quad \varepsilon \rightarrow 0,
$$

and in the Lévy approximation

$$
\left(g_{3}(\varepsilon)\right)^{-1} g_{1}(\varepsilon) f_{2}(\varepsilon) \rightarrow 1, \quad \varepsilon \rightarrow 0 .
$$

This normalization allows us to consider more processes and their small and large deviations. 


\section{REFERENCES}

1. H. Cramér, Sur un nouveau théorème-limite de la théorie des probabilités, Actualités Scientifiques et Industrielles 736 (1938), 5-23.

2. H. Chernoff, A measure of asymptotic efficiency for tests of a hypothesis based on the sum of observations, Ann. Math. Statist. 23 (1952), no. 4, 493-507.

3. J. Feng and T. G. Kurtz, Large Deviations for Stochastic Processes, Mathematical Surveys and Monographs, 131, AMS, Providence, RI, 2006

4. J. Jacod and A. N. Shiryaev, Limit Theorems for Stochastic Processes, Springer-Verlag, Berlin, 2003.

5. A. Puhalskii, On functional principle of large deviations, New Trends in Probability and Statistics, vol. 1, VSP, Utrecht, 1991, pp. 198219.

6. V. S. Korolyuk and I. V. Samoĭlenko, Large deviations problem for processes of random evolution, Teor. Imovir. Mat. Stat. 93 (2016), 88-95. (Ukrainian)

7. Ya. I. Yeleyko and O. A. Yarova, The behavior of the generator normalization factor in approximation of random processes, Cybernetics and Systems Analysis. 52 (2016), pp. 147-153.

8. O. A. Yarova, About selection a small normalization parameter for generator of random process, Visn. L'viv. Univ., Ser. Mekh.-Mat. 81 (2016), pp. 159-162.

9. Ya. I. Yeleyko and O. A. Yarova, The Problem of Large Deviations for Markov Evolutions in the Scheme of Poisson and Lévy Approximation, Columbia International Publishing. Contemporary Mathematics and Statistics. 4 (2017), pp. $28-40$. 